\title{
Perception of a single-session pre-in vitro fertilisation counselling service and attitudes towards support group: a survey of patients in Singapore
}

\author{
Gaik Suan $\underline{\mathrm{Lim}}^{1}$, MA, Reshmi Karayan $\underline{\text { Kayanoth }}^{1}$, MSc, MPhil, Birit FP $\underline{B r o e k m a n}^{2}$, MD, MSRc, Cornelia YI $\underline{\text { Chee }}^{1}$, MBBS, MMed
}

\begin{abstract}
INTRODUCTION This study aimed to investigate patients' evaluation of a compulsory pre-in vitro fertilisation (IVF) counselling session in Singapore and determine their attitudes towards attending a support group during IVF treatment. METHODS 464 patients due to undergo their first IVF treatment were recruited at the Clinic for Human Reproduction, National University Hospital, Singapore. Prior to IVF treatment, all patients attended a counselling session conducted by a clinical psychologist trained in infertility counselling. The Depression Anxiety Stress Scales 21 was used to measure patients' psychological symptoms of depression, anxiety and stress. A feedback form was administered after the session to determine their evaluation of the session and interest in attending a support group.

RESULTS After the pre-IVF counselling session, $90.9 \%$ of patients reported that the session was useful, with over $80 \%$ of participants reporting that the session had helped them to better prepare for the IVF treatment, enhanced their coping and enabled them to better communicate their needs to their spouse. Overall, $64.1 \%$ of patients expressed interest in attending a support group, with male patients showing more interest. Financial resources and the level of psychological symptoms experienced were found to influence patients' motivation to attend a support group.

CONCLUSION The single pre-IVF counselling session was well received and found to be useful by patients. Hence, it is recommended that IVF counselling be made an essential part of the holistic care given to patients undergoing IVF, particularly those who experience higher levels of distressing psychological symptoms.
\end{abstract}

Keywords: counselling, infertility counselling, IVF counselling

\section{INTRODUCTION}

Infertility counselling to assist couples who are undergoing in vitro fertilisation (IVF) to manage their stress during treatment is still not a common practice, especially in Asian countries. Only a few studies have investigated the effectiveness of seeking professional help or the view of the patient undergoing IVF in the Asian context. ${ }^{(1-3)}$ To the best of our knowledge, only one study has been conducted in Singapore thus far, in which Thia et $a^{(1)}$ reported that a majority of participants agreed that ongoing counselling should be part of IVF treatment. On the other hand, results of studies conducted in Western countries have indicated mixed findings. For example, de Klerk et $\mathrm{al}^{\left({ }^{(4)}\right.}$ reported that in Laffont and Edelmann's study, ${ }^{(5)}$ $75 \%$ of participants who had undergone at least one IVF attempt expressed a wish for pretreatment counselling and almost half of their participants requested counselling during treatment. On the contrary, Schmidt et al found that only $10 \%-20.8 \%$ of female patients felt that professional psychosocial services were important. ${ }^{(6)}$

The purpose of the present study was to assess patients' evaluation of the usefulness of a compulsory pre-IVF counselling session in Singapore and determine their attitudes towards attending a support group during IVF treatment.

\section{METHODS}

Patients due to undergo IVF were recruited from the Clinic for Human Reproduction, National University Hospital (NUH),
Singapore. From May 2013 to December 2014, a total of 311 couples were referred for a pre-IVF counselling session prior to their first IVF cycle. All patients filled out the Depression Anxiety Stress Scales $21^{(7)}$ (DASS-21) before the counselling session and were invited to complete a feedback form after the session.

DASS-21 is the shorter version of Lovibond and Lovibond's 1995 42-item self-report questionnaire that was designed to measure the negative emotional states of depression, anxiety and stress. ${ }^{(7)}$ DASS-21 is a well-established instrument with a Cronbach's alpha of 0.91, 0.84 and 0.90 for the depression, anxiety and stress subscales, respectively. Although this scale has not been validated locally, it has been translated and validated in other Asian countries that have a similar population of mixed ethnicities and Eastern cultures such as Malaysia, ${ }^{(8)}$ China, Japan and Hong Kong. ${ }^{(9,10)}$ Both English and Chinese versions of DASS-21 were made available for our study.

Participants were required to indicate the presence of symptoms over the previous week. Each DASS-21 subscale contained seven items and was scored on a 4-point scale (score range $0-3,0=$ 'did not apply to me at all' and $3=$ 'applied to me very much or most of the time over the past week'). The total score from each subscale was then multiplied by two. The minimum and maximum scores were 0 and 42, respectively. The final score of these subscales was then categorised as normal, mild, moderate, severe and extremely severe (Table I).

${ }^{1}$ Department of Psychological Medicine, National University Hospital, ${ }^{2}$ Singapore Institute for Clinical Sciences, Agency for Science, Technology and Research (A*STAR), Singapore Correspondence: Ms Lim Gaik Suan, Senior Clinical Psychologist, Department of Psychological Medicine, National University Hospital, 1E Kent Ridge Road, NUHS Tower Block, Level 9, Singapore 119228. gaik_suan_lim@nuhs.edu.sg 
Table I. DASS-21 ${ }^{(7)}$ recommended cut-off scores for each subscale.

\begin{tabular}{|llll|}
\hline Score category & \multicolumn{3}{c|}{ Cut-off score } \\
\cline { 2 - 4 } & Depression & Anxiety & Stress \\
\hline Normal & $0-9$ & $0-7$ & $0-14$ \\
\hline Mild & $10-13$ & $8-9$ & $15-18$ \\
\hline Moderate & $14-20$ & $10-14$ & $19-25$ \\
\hline Severe & $21-27$ & $15-19$ & $26-33$ \\
\hline Extremely severe & $28+$ & $20+$ & $34+$ \\
\hline
\end{tabular}

DASS-21: Depression Anxiety Stress Scales 21

At NUH during the study period, the pre-IVF counselling session was a requirement for patients before they underwent their first IVF cycle. The counselling session was usually conducted on the same day as the first appointment with the IVF clinic nursing staff who had officially enrolled the patients into IVF treatment. The clinical psychologists who conducted the session were trained in infertility counselling by the British Infertility Counselling Association. Patients in the study received a 45-minute pre-IVF counselling session prior to beginning their IVF treatment. They were given the DASS-21 to be completed independently before the counselling session, which the clinical psychologist scored after the session.

The pre-IVF counselling session was a semistructured clinical interview session. Its broad focus was on risk assessment and psychoeducation. The session covered individual and family psychiatric history, marital and sexual history, social support network, stress management and coping strategies, reproductive history, feelings and responses towards infertility and treatment, attitude and anxiety level about IVF treatment and procedure, understanding of the emotional responses to infertility and its treatment, expectations regarding treatment outcome, possible challenges in ending treatment and adapting to a childless future/ alternative options. Based on the clinical psychologist's clinical assessment, appropriate strategies and considerations were shared with patients throughout the course of the session. When there was a need for follow-up and patients were keen, either a regular follow-up session with the clinical psychologist was scheduled, or an external referral to a psychiatrist, family therapist and/or sex therapist was suggested to best match the needs of the patients. Patients who declined follow-up or who were assessed not to be at risk were briefed about service availability, so that they could reconsider if they changed their minds or required the service in future.

At the end of the counselling session, all patients were invited to fill out a feedback form consisting of six statements, using a 5-point Likert scale (Box 1). The first five statements were designed to determine patients' evaluation of the usefulness of the session and their view of the therapist. The last statement was for patients to indicate their interest in attending a support group. Patients had to complete the feedback form independently at the waiting area without discussing it with their spouse and hand it to the counter staff at the clinic.

Data was analysed using SPSS version 13.0 (SPSS Inc, Chicago, IL, USA). $t$-test groups were used to explore significant

\section{Box 1. Questions on the feedback form.}

Q1 Attending the session helped me to better prepare myself for IVF (in vitro fertilisation) treatment and the procedure.

Q2 Attending the session helped me to prepare myself on how to cope better.

Q3 Attending the session helped me to better communicate my needs to my spouse/support my spouse better.

Q4

Generally, I found that attending today's session was useful.

Q5 The therapist was supportive.

Q6 If there is a support group for those undergoing IVF, I would be keen to attend.

differences between genders. One-way analysis of variance (ANOVA) was used to compare variables such as educational background, employment status, personal income, nationality and DASS-21 scores. Tukey's HSD (honest significant difference) post-hoc test was used when a significant difference was indicated among the groups by one-way ANOVA. A p-value $<0.05$ was considered to be statistically significant.

\section{RESULTS}

Out of a total of 622 referred patients, 505 (81.2\%) patients completed the surveys. After excluding non-first-timer IVF patients, only 464 patients were included in the analysis. Table II shows the sociodemographic profile of the patients. A majority of the patients were aged 38-39 years (women $41.7 \%$, men $35.3 \%$ ) and $40-42$ years (women $32.3 \%$, men $36.2 \%$ ). Most were of Chinese ethnicity (women $70.7 \%$, men $65.8 \%$ ), had attained at least a degree or higher qualification (women $71.0 \%$, men $68.5 \%$ ), were employed (women $80.1 \%$, men $86.4 \%$ ) and were Singapore citizens (women $65.3 \%$, men $71.9 \%$ ).

$6.8 \%$ of the patients reported having at least moderate levels of depressive symptoms, $16.2 \%$ reported moderate symptoms of anxiety and $5.0 \%$ reported moderate symptoms of stress. Women scored significantly higher on all subscales as compared to their spouses (depression subscale: $t[454]=1.874, p=0.001$; anxiety subscale: $t$ [454] $=4.179, \mathrm{p}=0.000$; stress subscale: $t[452]=-2.855, \mathrm{p}=0.000)$.

A majority of patients gave very positive feedback about the compulsory single pre-IVF counselling session. $88.9 \%$ of patients (women $88.2 \%$, men $89.6 \%$ ) found that attending the session helped them to better prepare for the IVF treatment and procedure. $89.6 \%$ of patients (women $88.7 \%$, men $90.6 \%$ ) reported that attending the session helped them to better cope while preparing for the IVF treatment. $86.4 \%$ of patients (women $84.1 \%$, men $88.8 \%$ ) indicated that the session helped them to better communicate their needs to their spouse and/or support their spouse. Sociodemographic factors, such as gender, educational background, employment status, individual personal income and nationality, as well as DASS-21 scores, were not associated with patients' evaluation of the usefulness of the session.

90.9\% of patients (women 90.4\%, men 91.4\%) reported that attending the session was useful. Again, sociodemographic factors, such as gender, educational background, employment 
Table II. Participants' sociodemographic profile.

\begin{tabular}{|c|c|c|}
\hline \multirow[t]{2}{*}{ Variable } & \multicolumn{2}{|c|}{ No. (\%) } \\
\hline & Female & Male \\
\hline \multicolumn{3}{|l|}{ Age (yr) } \\
\hline$<35$ & $3(1.3)$ & $1(0.5)$ \\
\hline $35-37$ & 49 (20.9) & $12(5.5)$ \\
\hline $38-39$ & $98(41.7)$ & $77(35.3)$ \\
\hline $40-42$ & $76(32.3)$ & $79(36.2)$ \\
\hline$>42$ & $9(3.8)$ & $49(22.5)$ \\
\hline Total & 235 & 218 \\
\hline \multicolumn{3}{|l|}{ Ethnicity } \\
\hline Chinese & $169(70.7)$ & $146(65.8)$ \\
\hline Malay & $16(6.7)$ & $17(7.7)$ \\
\hline Indian & $36(15.1)$ & $38(17.1)$ \\
\hline Other & $18(7.5)$ & $21(9.5)$ \\
\hline Total & 239 & 222 \\
\hline \multicolumn{3}{|l|}{ Educational background } \\
\hline Primary school & $1(0.4)$ & $3(1.4)$ \\
\hline Secondary school & $17(7.1)$ & $22(9.9)$ \\
\hline Junior college/diploma & $51(21.4)$ & $45(20.3)$ \\
\hline Degree & $120(50.4)$ & $97(43.7)$ \\
\hline Postgraduate and above & $49(20.6)$ & $55(24.8)$ \\
\hline Total & 238 & 222 \\
\hline \multicolumn{3}{|l|}{ Employment status } \\
\hline Unemployed & $21(8.9)$ & $1(0.5)$ \\
\hline Self-employed & $15(6.4)$ & $27(12.2)$ \\
\hline Employed & $189(80.1)$ & $191(86.4)$ \\
\hline Other & $11(4.7)$ & $2(0.9)$ \\
\hline Total & 236 & 221 \\
\hline \multicolumn{3}{|l|}{ Personal income (SGD) } \\
\hline$<1,500$ & $57(26.3)$ & $24(11.1)$ \\
\hline $1,500-2,999$ & $16(7.4)$ & $9(4.1)$ \\
\hline $3,000-4,499$ & $48(22.1)$ & $45(20.7)$ \\
\hline $4,500-5,999$ & $42(19.4)$ & $50(23.0)$ \\
\hline $6,000-7,499$ & $18(8.3)$ & $20(9.2)$ \\
\hline $7,500-9,999$ & $16(7.4)$ & $24(11.1)$ \\
\hline$>10,000$ & $20(9.2)$ & $45(20.7)$ \\
\hline Total & 217 & 217 \\
\hline \multicolumn{3}{|l|}{ Nationality } \\
\hline Singapore citizen & $156(65.3)$ & $159(71.9)$ \\
\hline Permanent resident & $56(23.4)$ & $42(19.0)$ \\
\hline Other & $27(11.3)$ & $20(9.0)$ \\
\hline Total & 239 & 221 \\
\hline
\end{tabular}

Total no. for each variable differs due to missing data.

status and personal income level, as well as DASS-21 scores, were not associated with these evaluations, except for nationality. Post-hoc analysis revealed that non-Singaporean patients rated the usefulness of the session more positively when compared to their Singaporean counterparts $(F[2,456]=4.427 ; p=0.012)$.

Regarding their view of the therapist, $96.5 \%$ of patients (women 96.6\%, men 96.4\%) strongly agreed or agreed that the therapist was supportive. Again, non-Singaporean patients rated the therapist as more supportive compared to their Singaporean counterparts $(F[2,458]=3.126 ; \mathrm{p}=0.045)$. Other variables, such as gender, educational background, employment status, personal income and DASS-21 scores, were not associated with patients' views of the therapist.

$64.1 \%$ of patients indicated that they were keen to attend a support group. Men showed more interest in attending the support group when compared to women $(t[430]=1.206 ; \mathrm{p}=0.018)$. Personal income level was found to significantly influence patients' interest in attending a support group. Post-hoc analysis indicated that patients with a personal income of SGD 4,500-5,999 were less keen to attend the support group when compared to those with a personal income level of SGD 6,000-7,499 $(F[6,403]=2.307 ; p=0.033)$. No difference was found among patients with other personal income levels. Patients' DASS-21 score was also found to play an important role in determining their interest in attending the support group. Posthoc analysis revealed that patients who scored at a moderate level of distress and above on at least one DASS- 21 subscale showed more interest in attending the support group $(t[428]=1.374 ; p=0.002)$. Other sociodemographic factors, such as educational background, employment status and nationality, were not associated with interest in attending the support group.

\section{DISCUSSION}

A majority of our patients favourably rated the usefulness of the pre-IVF counselling session. Their satisfaction level with regard to its usefulness was $81.4 \%-91.4 \%$. This was consistent with another local study ${ }^{(1)}$ in which $80 \%$ of respondents found that psychosocial counselling helped them develop coping strategies and enabled them to cope better with an unfavourable outcome of treatment. These findings suggest that even a brief intervention, such as a single pre-IVF counselling session, would benefit patients undergoing IVF treatment.

In our study, except for nationality, other sociodemographic factors such as gender, educational background, employment status and personal income, as well as DASS-21 scores, were not associated with patients' evaluations of the usefulness of the session. Non-Singaporean patients rated more highly the usefulness of pre-IVF counselling and their therapist's supportiveness when compared to Singaporean patients. The relationship between patients' social backgrounds and their satisfaction with services had mixed results, similar to other available studies. For instance, a study by Sabourine et al in 1991, as reported by Schmidt et al, ${ }^{(11)}$ showed that patients' education level and financial status do not have significant influence on their evaluations of satisfaction with fertility treatments. On the other hand, Hall and Dornan ${ }^{(11)}$ and Schmidt et $\mathrm{al}^{(11)}$ found that patients with lower socioeconomic status viewed medical staff with more reverence, and hence were more reluctant to give negative evaluations when compared to patients from higher social classes.

In our study, the difference in patients' evaluations of the overall usefulness of the session and the therapist's supportiveness was only observed between non-Singaporean and Singaporean patients. A majority ( $n=103 / 145,71.0 \%$ ) of non-Singaporean patients were from neighbouring Asian countries and culturally 
may have more reverence for healthcare professionals, as observed by Schmidt et al. ${ }^{(11)}$ The fact that these patients were away from their home country and extended families, which are traditionally an important source of support among Asian families, could also have played an important role in influencing patients' evaluation of the service provided.

The high interest expressed in receiving further support $(64.1 \%)$ was consistent with the results of many other studies that have reported interest in the range of $48 \%-90 \% .^{(12,13)}$ Laffont and Edelmann ${ }^{(5)}$ indicated that patients feel reassured to know that counselling is available and view counselling as a service that should be offered as part of fertility treatment, even if they do not intend to utilise the service. As Boivin et $\mathrm{al}^{(12)}$ also reported, studies showed that many IVF patients were dissatisfied with the lack of psychosocial support services offered before, during and/or after treatment by IVF centres. These findings in various studies underscore that IVF patients recognise the benefit of and need for infertility counselling and feel reassured by the knowledge that it is available. We agree with Boivin et $\mathrm{al}^{(12)}$ that the relatively low take-up rate of the service should not be interpreted as a lack of interest in psychosocial support services, especially when patients have never experienced it, and that infertility counselling should be part of the overall care accorded to IVF patients.

In future studies and service planning, more attention should be paid to what may be hindering or preventing patients from using the service. To increase take-up rates, we may wish to implement the recommendations of researchers such as Goodman and Rothman and Pengelly et al, as reported by Boivin et $\mathrm{al}_{,}{ }^{(12)}$ to recruit patients using more personalised approaches, including face-to-face sessions, instead of through notices or letters. Connolly et al and Hernon et al have also demonstrated that patients generally find counselling beneficial after they have been persuaded to attend a session. ${ }^{(12)}$ This indicates that patients may lack knowledge or be unsure about the potential benefits of the counselling service; hence, their expressions of interest or willingness to receive the service should be interpreted with caution. Apart from this, these authors also found that patients generally felt awkward about contacting a counsellor, were concerned about their privacy, feared that they may be perceived as emotionally and/or mentally abnormal, and had practical concerns such as the cost and time investment involved in scheduling additional sessions on top of existing medical appointments. Several other studies have also suggested that patients may be reluctant to seek mental health services out of fear of stigmatisation. ${ }^{(14-16)}$ In view of this, working closely with the medical team and including IVF counselling as a part of routine IVF treatment is of utmost importance. The latter would curb patients' fears of being stigmatised and offer a less threatening way to introduce the service. This would be a more subtle way to educate patients that IVF counselling is an essential part of their holistic care instead of being an additional service that serves a problematic or abnormal population.

An interesting finding from our study was that men showed more interest in attending a support group even when they were compared to women who had higher scores on all DASS-21 subscales. This was contrary to studies by Boivin et $\mathrm{al}^{(12)}$ and Glazer et al, as reported by Covington and Burns, ${ }^{(15)}$ in which women were found to be more willing to attend a support group and used significantly more sources of support than men. Our finding is important in light of past research showing that patients who were distressed and wanted to consult a counsellor did not do so for practical reasons, such as the perceived difficulty of scheduling sessions. ${ }^{(5)}$ While it is understandable that women would already have numerous medical appointments related to IVF treatment, their reasons for not wishing to attend a support group could be a critical question to study in the future, such as to ascertain whether this was due to time constraints, the perceived need for IVF counselling or a perception that the amount of informal support that they received was sufficient. Attending a support group may have been less taxing for men due to the relatively fewer medical appointments that they were required to attend, resulting in the finding that men were more keen to attend counselling sessions. However, we were not able to ascertain the motivations behind their greater interest in attending a support group compared to women, as some men accompany their partners for every medical appointment. Hence, understanding their needs and expectations from IVF counselling is necessary in order to best cater to their needs.

Apart from this, gender roles in society at large might have also influenced attitudes towards support groups among our patients. Although Singapore is a developed country, some Singaporeans still have conservative expectations regarding gender roles, expecting men to play a supportive role and seeing it as less acceptable for them to express weakness and/or emotional needs. This expectation may make it more challenging for male patients to receive the support that they need from their social network, hence making them more receptive to formal support when it is made available. As we did not explore the causes of infertility in our study, we are uncertain if these may also have played a part in determining how comfortable patients were in seeking support from their social circles when they had to divulge their difficulties.

Patients from the middle income group (SGD 4,500-5,999) were less keen to attend a support group when compared to those with higher personal incomes (SGD 6,000-7,499). However, this difference was not observed across other personal income groups (e.g. lower vs. higher income group). We believe that although financial standing could be one of the influencing factors, perceived financial resources was another significant consideration for some patients. A significant number of patients said that they used online forums or chat rooms for information and emotional support, further validating the finding from Cousineau and Domar's study ${ }^{(14)}$ that more than half of their patients went online to gather fertility-related information regardless of their socioeconomic status. This suggests a need to design more practical and cost-effective ways of providing support, such as through printout material, or online information and services that may be less financially taxing for patients on top of the medical costs of their IVF treatment.

Our findings supported the views of Boivin et $\mathrm{al}^{(12)}$ and Wischmann et $\mathrm{al}^{(17)}$ that patients with a higher level of distress 
were more likely to use counselling services. In our study, patients who scored at a moderate level and above on at least one DASS21 subscale expressed more interest in attending a support group. This demonstrated that patients' experience of a higher DASS-21 score was still a useful predictor of their willingness to attend a support group, in keeping with the findings of Boivin et $\mathrm{al}^{(12)}$ and de Klerk et al, ${ }^{(4)}$ who observed that a majority of infertility patients did not consider themselves to be distressed enough to need counselling. These authors suggested that patients seek psychological treatment not because they experience distress, but because they cannot manage it.

To the best of our knowledge, the present study is one of the first few to have examined factors that may influence patients' interest in receiving infertility counselling in the Asian context. It had a high participation rate of $81.2 \%$, which indicates that we surveyed a majority of the clinic's population and that our findings may be generalised to local clinical samples. Nevertheless, it would be useful to obtain the opinions of the non-respondents, who accounted for $18.8 \%$ of our patient population, as the focus of a future study.

There were several limitations to our study. First, DASS-21 is a general self-reported questionnaire that measures patients' negative emotional states with regard to their depression, anxiety and stress levels. Although it may indicate symptoms, the instrument is not the ideal tool to assess participants' mental well-being, specifically in relation to IVF treatment, and does not provide any clinical diagnosis. Furthermore, we did not conduct any reassessments over time, as follow-up or monitoring, of participants' coping and mental well-being after single-session counselling was performed and throughout their IVF treatment period. Follow-up sessions and periodical screening could be used to monitor patients' distress and coping, especially after crucial milestones in treatment, such as after embryo transfer, during the waiting period and even following failed treatment.

Another limitation was that we did not explore other important variables, such as the causes and duration of infertility and their influence on other variables in the study. Hakim et $\mathrm{al}^{(2)}$ have shown that patients with primary infertility expected that counselling would be more informative and more helpful than patients with secondary infertility. Hence, it is important to look into this aspect in future studies.

In addition, we did not track how many assessed at-risk patients had turned down the offer for a follow-up session and hence were unable to provide accurate take-up rates. Furthermore, in the absence of continued and/or long-term follow-up, we did not have information on the patients' mental well-being during and after IVF treatment. Future studies could also explore the effects of counselling sessions and support groups on symptoms of depression, anxiety and stress through a comparison of scores before and after these sessions. Long-term follow-up studies on IVF patients' mental well-being would provide more valuable and convincing insights for future service development.

Lastly, other psychosocial factors such as financial burden, personal and/or marital distress, the need to strike a balance between work commitments and medical treatment, as well as social support, may have contributed to patients' attitudes towards the counselling service. It would be crucial to explore these in future studies. A more comprehensive study design that takes into account all of these variables would help to provide insight into outstanding queries that have not been addressed in our study. A larger sample size and longitudinal follow-up would enable such comprehensive analysis.

To our knowledge, our study is the first in Asia that looked beyond patient satisfaction level in IVF counselling and explored factors that may influence patients' interest in receiving psychological intervention. The study demonstrated that a single pre-IVF counselling session was well received by patients as they found it useful in preparing them to better cope and communicate with their partners when they embark on IVF treatment. Hence, it is recommended that IVF counselling be made an essential part of the holistic care given to patients undergoing IVF, particularly for those who experience higher levels of distressing psychological symptoms. One interesting finding that contradicted those from other studies was that our male patients were more willing to attend support groups than their female counterparts, although the latter group reported more severe psychological symptoms. Two other factors we identified that influenced patients' interest in receiving psychological intervention were their personal income level and the degree of psychological symptoms they experienced. These findings can be used by providers of IVF for service development and implementation strategies to improve the well-being of patients undergoing IVF.

\section{ACKNOWLEDGEMENTS}

We gratefully acknowledge our study's participants and the help of the staff at the Clinic for Human Reproduction, NUH, Singapore, in diligently distributing and collecting the DASS-21 and feedback forms from participants. We are also deeply grateful for the support of Prof PC Wong and his team of clinicians in referring patients to our service. Last but not least, thanks are due to Sharon and Rachael, our colleagues from NUH Women Emotional Health Services, for helping with data entry and analysis.

\section{REFERENCES}

1. Thia EW, Vo Thanh LA, Loh SK. Study on psychosocial aspects and support of in vitro fertilisation programme in an Asian population. Singapore Med J 2007; 48:61-8.

2. Hakim LZ, Newton CR, Maclean-Brien D, Feyles V. Evaluation of preparatory psychosocial counselling for medically assisted reproduction. Hum Reprod 2012; 27:2058-66.

3. Jin X, Wang G, Liu S, et al. Survey of the situation of infertile women seeking in vitro fertilization treatment in China. Biomed Res Int 2013; 2013:179098.

4. de Klerk C, Hunfeld JA, Duivenvoorden HJ, et al. Effectiveness of a psychosocial counselling intervention for first-time IVF couples: a randomized controlled trial. Hum Reprod 2005; 20:1333-8.

5. Laffont I, Edelmann RJ. Perceived support and counselling needs in relation to in vitro fertilization. J Pyschosom Obstet Gynaecol 1994; 15:183-8.

6. Schmidt L, Holstein BE, Boivin J, et al. Patients' attitudes to medical and psychosocial aspects of care in fertility clinics: findings from the Copenhagen Multi-centre Psychosocial Infertility (COMPI) Research Programme. Hum Reprod 2003; 18:628-37.

7. Lovibond SH, Lovibond PF. Manual for the Depression Anxiety Stress Scales. 2nd ed. Sydney: Psychology Foundation, 1995.

8. Musa R, Ramli R, Abdullah K, Sarkarsi R. Concurrent validity of the depression and Anxiety components in the Bahasa Malaysia version of the Depression Anxiety and Stress Scales (DASS). ASEAN J Psychiatry 2011; 12 
9. Moussa MT, Lovibond PF, Laube R. Psychometric properties of a Chinese version of the short Depression Anxiety Stress Scales (DASS21). Report for New South Wales Transcultural Mental Health Centre, Cumberland Hospital, Sydney. 2001

10. Chan RC, Xu T, Huang J, et al. Extending the utility of the Depression Anxiety Stress scale by examining its psychometric properties in Chinese settings. Psychiatry Res 2012; 200:879-83.

11. Schmidt L, Holstein BE, Boivin J, et al. High ratings of satisfaction with fertility treatment are common: findings from the Copenhagen Multi-centre Psychosocial Infertility (COMPI) Research Programme. Hum Reprod 2003; 18:2638-46.

12. Boivin J, Scanlan LC, Walker SM. Why are infertile patients not using psychosocial counselling? Hum Reprod 1999; 14:1384-91.
13. Hammarberg K, Astbury J, Baker H. Women's experience of IVF: a follow-up study. Hum Reprod 2001; 16:374-83.

14. Cousineau TM, Domar AD. Psychological impact of infertility. Best Pract Res Clin Obstet Gynaecol 2007; 21:293-308.

15. Covington SN, Burns LM. Infertility Counseling: A Comprehensive Handbook for Clinicians. 2nd ed. Cambridge: Cambridge University Press, 2006.

16. Vogel DL, Wester SR, Larson LM. Avoidance of counseling: psychological factors that inhibit seeking help. J Couns Dev 2007; 85:410-22.

17. Wischmann T, Scherg H, Strowitzki T, Verres R. Psychosocial characteristics of women and men attending infertility counselling. Hum Reprod 2009; 24: 378-85. 